\title{
Cardioprotective Effects of Cysteine Alone or in Combination With Taurine in Diabetes
}

\author{
P. S. TAPPIA ${ }^{1}$, Y-J. XU ${ }^{2}$, D. RODRIGUEZ-LEYVA ${ }^{2}$, N. AROUTIOUNOVA ${ }^{2}$, \\ N. S. DHALLA ${ }^{2}$ \\ ${ }^{1}$ Asper Clinical Research Institute, St. Boniface Hospital Research, Winnipeg, Manitoba, Canada, \\ ${ }^{2}$ Institute of Cardiovascular Sciences, Department of Physiology, Faculty of Medicine, University \\ of Manitoba, Winnipeg, Canada
}

Received May 18, 2012

Accepted November 2, 2012

On-line December 13, 2012

\section{Summary}

This study was undertaken to examine the effects of dietary supplementation of cysteine and taurine in rats with diabetes induced with streptozotocin (STZ, $65 \mathrm{mg} / \mathrm{kg}$ body weight). Experimental animals were treated orally (by gavage) with cysteine $(200 \mathrm{mg} / \mathrm{kg}$ ) and taurine $(400 \mathrm{mg} / \mathrm{kg})$, alone or in combination, daily for 8 weeks. In one group, rats were also pretreated 3 weeks before the induction of diabetes (prevention arm) whereas in the other, the treatment was started 3 days after the induction of diabetes (reversal arm). Diabetes increased heart weight/body weight (HW/BW) ratio, plasma glucose, triglyceride and cholesterol levels as well as depressed heart rate (HR), blood pressure, left ventricular systolic pressure (LVSP), rate of contraction (+dP/dt), rate of relaxation (-dP/dt), fractional shortening (FS) and cardiac output (CO). The left ventricular internal diameter in systole (LViDs) was increased whereas that in diastole (LViDd) was decreased. In the prevention arm, treatment of the diabetic animals with cysteine or taurine decreased HW/BW ratio and improved $\mathrm{HR}, \mathrm{FS},+\mathrm{dP} / \mathrm{dt}$ and $-\mathrm{dP} / \mathrm{dt}$, as well as normalized LViDs, without altering the increase in glucose level. Cysteine decreased plasma triglyceride and cholesterol levels and improved LVSP whereas $\mathrm{CO}$ was improved by taurine. In the reversal arm, cysteine alone or with taurine did not correct the changes in hemodynamic parameters, FS and plasma triglycerides. Diabetes-induced cardiac dysfunction and increases in plasma triglycerides can be prevented, but not reversed, by dietary cysteine alone or in combination with taurine.

\section{Key words}

Diabetes • Cardiac dysfunction • Lipid profile • Blood glucose • Cysteine supplementation $\bullet$ Taurine supplementation

\section{Corresponding author}

P. S. Tappia, Asper Clinical Research Institute, CR3129-369 Tache Avenue, Winnipeg, Manitoba, Canada R2H 2A6. Fax: 204235-0793. E-mail: ptappia@sbrc.ca

\section{Introduction}

Hyperglycemia is known to result in overproduction of reactive oxygen species (Du et al. 2003). It is now believed that oxidative stress associated with deficit in the status of the antioxidant defense plays a critical role in subcellular remodeling, $\mathrm{Ca}^{2+}$-handling abnormalities and subsequent cardiac dysfunction and vascular defects during diabetes (Dhalla et al. 1998). Glutathione is the most abundant low molecular weight thiol and the ratio of reduced glutathione to oxidized glutathione is the major redox couple the deficiency of which contributes to oxidative stress and pathogenesis of diabetes ( $\mathrm{Wu}$ et al. 2004). Cysteine availability is considered as a major rate limiting factor in glutathione production (Meister et al. 1986, Griffith 1999). Sources of cysteine in mammals are dietary protein, endogenous proteolysis and conversion via methionine (O'Connor et al. 1995). In fact, cellular levels of cysteine and glutathione have been reported to be reduced in human diabetics (Sekhar et al. 2011). The cardioprotective effects of cysteine are considered to be due to the direct scavenging of free radicals and/or improved antioxidant capacity through glutathione preservation (Shackebaei et al. 2005).

The beneficial actions of taurine, which is also 
a sulfur-containing amino acid, on the cardiovascular system include protection against ischemia-reperfusion injury, modulation of intracellular calcium concentration $\left(\left[\mathrm{Ca}^{2+}\right]_{\mathrm{i}}\right)$ as well as antioxidant and antiatherogenic effects (Xu et al. 2008). Although the biosynthetic capacity of taurine is very low in humans and the diet is its major source in the body (Hansen 2001), taurine can be synthesized from cysteine (Bouckenooghe et al. 2006). In addition, intracellular taurine is depleted during the development of diabetes (Hansen 2001). We have earlier reported that dietary supplementation with taurine of streptozotocin (STZ)-induced diabetic rats attenuated the diabetes-induced abnormalities in cardiac contractile function and ultrastructure (Tappia et al. 2011); however, no information regarding the beneficial effects of cysteine on heart function in diabetes is available in the literature.

This study was undertaken to test if cysteine exerts any beneficial effects on diabetes-induced cardiac dysfunction. Furthermore, treatment of diabetic animals with taurine was carried out to examine the comparative actions of cysteine and taurine in diabetic animals. Experiments were also conducted to investigate whether treatment of diabetic animals with a combination of cysteine and taurine produces any additive effects. In addition to studying the actions of cysteine and/or taurine on diabetes-induced hemodynamic alterations and cardiac remodeling, changes in plasma glucose and lipid levels were also monitored. The preventive effects of both cysteine and taurine were evaluated in animals pretreated with these amino acids before the induction of diabetes whereas the therapeutic effects of these interventions were examined upon starting treatments after inducing diabetes.

\section{Methods}

\section{Diabetes model}

The use of animals and protocols in this study was approved by the University of Manitoba Animal Care Committee in accordance with the guidelines of the Canadian Council on Animal Care and the Guide for the Care and Use of Laboratory Animals (1985), NIH,
Bethesda. Male Sprague-Dawley (225-250 g) rats were made diabetic with a single tail vein injection of streptozotocin (STZ) $(65 \mathrm{mg} / \mathrm{kg}$ body weight, dissolved in $0.1 \mathrm{M}$ citrate buffer, $\mathrm{pH} 4.5$ ) as described previously (Tappia et al. 2004, 2011). The STZ-induced diabetes in rats is a well-established model that resembles human type 1 diabetes. Age-matched normal animals received citrate buffer and served as controls; all rats were fed ad libitum. Cysteine; $200 \mathrm{mg} / \mathrm{kg}$ body weight (BW) (Bae et al. 2005) and taurine; $400 \mathrm{mg} / \mathrm{kg} \mathrm{BW}$ (Tappia et al. 2011) either alone or in combination, were administered daily (by oral gavage) for 8 weeks. In one set of experiments, rats were pre-treated 3 weeks before the induction of diabetes with STZ and the treatment was continued for a further 8 weeks (prevention arm). In another set of experiments, treatment of rats with these amino acids was started 3 days after inducing diabetes with STZ (reversal arm). Control and non-treated diabetic animals were subjected to the same handling and were given water (vehicle) alone by oral gavage. Diabetes was confirmed by measurement of glucose in the urine $>55 \mathrm{mM}$ (Diastix reagent test strips for glucose, Bayer Inc., Toronto, ON, Canada). Blood samples from diabetic animals treated with and without amino acids individually or in combination were taken at the time of sacrifice ( 8 weeks post-STZ) and analyzed for plasma glucose levels, total cholesterol, triglycerides, and high density lipoprotein (HDL) cholesterol levels using standard methods by Laboratory Services at St. Boniface Hospital operated by Diagnostic Services of Manitoba. In some experiments, insulin levels in plasma were analyzed by standard methods.

Echocardiographic and cardiac performance assessment An ultrasound imaging system (SONOS 5500 ultrasonograph; Agilent Technologies, Mississauga, ON, Canada) was used to measure heart rate (HR), systolic and diastolic dimensions as described previously (Tappia et al. 2004). Cardiac output (CO) and fractional shortening (FS) were calculated using the following formula:

$$
\begin{aligned}
& \mathrm{CO}(\mathrm{l} / \mathrm{min})=\text { stroke volume } \mathrm{x} \text { heart rate } / 1000 \\
& \mathrm{FS}(\%)=\frac{\text { LV end }- \text { diastolic dimension }(\mathrm{mm}) \mathrm{LV} \text { end }- \text { systolic dimension }(\mathrm{mm}) \times 100}{\text { LV end }- \text { diastolic dimension }(\mathrm{mm})}
\end{aligned}
$$


The left ventricle (LV) posterior wall thickness (PWT) and internal diameters during systole (LViDs) and diastole (LViDd) were determined to gain information regarding cardiac remodeling in diabetic animals treated with and without amino acids. After echocardiographic examination and just before the animal sacrifice, cardiac performance assessment as conducted by the catheterization technique described elsewhere (Tappia et al. 2011). Cardiac performance data was computed and displayed using AcqKnowledge Software version 3.7.1 (MP System “Quick Start”, Biopac System, Inc., Goleta, CA, USA). Thus the same animals in each group were used for echocardiographic and hemodynamic assessment as well as plasma analysis.

\section{Statistical analysis}

Microcal Origin version 7.5 (Origin Lab Corp., Northampton, MA, USA) was used for statistical analysis of the data. All values are expressed as mean \pm SEM. The differences among all groups were evaluated by one-way ANOVA followed by Student's t-test for comparisons between two groups. A probability of $95 \%$ or more $(\mathrm{P}<0.05)$ was considered significant.

\section{Results}

General characteristics of diabetic animals treated with cysteine and taurine

Table 1 shows that the BW of the diabetic animals was significantly lower than the controls, whereas a significant increase in the heart weight (HW) to body weight (HW/BW) ratio, an index of cardiac hypertrophy, was seen in the diabetic animals. In the pretreated animals, cysteine and taurine alone or in combination prevented the diabetes-induced increase in $\mathrm{HW} / \mathrm{BW}$ ratio without affecting depressions in body, heart or ventricular weights. In contrast, when the treatment with cysteine and taurine alone or in combination were started 3 days after inducing diabetes, the diabetes-induced alterations in BW, HW and HW/BW ratio were not reversed (Table 1). Diabetes resulted in elevations in the plasma levels of glucose, triglycerides and total cholesterol without any changes in HDLcholesterol (Table 2). For the prevention protocol, treatment with cysteine, unlike taurine, decreased these changes in triglycerides and cholesterol without affecting the glucose levels in the diabetic animals; no additive effect of the combination of cysteine with taurine was observed. For the reversal protocol, treatment with cysteine and taurine alone or in combination did not affect the diabetes-induced increases in plasma glucose, triglycerides and total cholesterol (Table 2). Although diabetes resulted in a significant reduction in the plasma concentration of insulin, the level of insulin was partially normalized with cysteine while no effect on insulin was seen with taurine or combination of cysteine and taurine in the prevention arm. Insulin levels in plasma of diabetic animals in the reversal arm were not determined. No changes in BW and $\mathrm{HW}$ as well as glucose and triglycerides levels were seen in the cysteine pre-treated

Table 1. General characteristics of diabetic rats treated with cysteine $(200 \mathrm{mg} / \mathrm{kg})$ or taurine $(400 \mathrm{mg} / \mathrm{kg})$, alone or in combination for a period of 8 weeks.

\begin{tabular}{llllll}
\hline & BW $(\mathbf{g})$ & HW $(\mathbf{m g})$ & LVW $(\mathbf{m g})$ & RVW $(\mathbf{m g})$ & HW/BW ratio (mg/g) \\
\hline $\begin{array}{l}\text { Control } \\
\text { Pre-treatment }\end{array}$ & $561 \pm 19$ & $1586 \pm 33$ & $1037 \pm 27$ & $284 \pm 19$ & $2.8 \pm 0.1$ \\
$\begin{array}{l}\text { Diabetes } \\
\text { Diabetes }+ \text { cysteine }\end{array}$ & $402 \pm 11^{*}$ & $1314 \pm 32^{*}$ & $916 \pm 12^{*}$ & $206 \pm 21^{*}$ & $3.3 \pm 0.1^{*}$ \\
$\begin{array}{l}\text { Diabetes }+ \text { taurine } \\
\text { Diabetes }+ \text { combination }\end{array}$ & $417 \pm 16$ & $1220 \pm 74$ & $818 \pm 21 \#$ & $252 \pm 19 \#$ & $2.9 \pm 0.2 \#$ \\
Post-treatment & $393 \pm 14$ & $1145 \pm 82$ & $760 \pm 32 \#$ & $211 \pm 12$ & $2.9 \pm 0.2 \#$ \\
$\begin{array}{l}\text { Diabetes } \\
\text { Diabetes }+ \text { cysteine }\end{array}$ & $389 \pm 32 *$ & $1222 \pm 97 *$ & $797 \pm 54 *$ & $223 \pm 31 *$ & $3.1 \pm 0.1 *$ \\
$\begin{array}{l}\text { Diabetes }+ \text { taurine } \\
\text { Diabetes }+ \text { combination }\end{array}$ & $340 \pm 21$ & $1054 \pm 58$ & $669 \pm 40 \#$ & $192 \pm 12$ & $3.1 \pm 0.1$ \\
\hline
\end{tabular}

Data are mean \pm SE of 5-9 animals for each group. Pre-treated animals also received 3 weeks treatment prior to the induction of diabetes whereas post-treated animals were given treatment 3 days after the induction of diabetes. BW, body weight; HW, heart weight; LVW, left ventricular weight; RVW, right ventricular weight. * $\mathrm{P}<0.05$ vs. control value; \# $\mathrm{P}<0.05$ vs. un-treated diabetic value. 
Table 2. Effects of cysteine $(200 \mathrm{mg} / \mathrm{kg})$ and taurine $(400 \mathrm{mg} / \mathrm{kg})$ alone or in combination on plasma glucose, lipid profile and insulin level in diabetic rats for a period of 8 weeks.

\begin{tabular}{|c|c|c|c|c|c|}
\hline & $\begin{array}{c}\text { Glucose } \\
\text { (mM) }\end{array}$ & $\begin{array}{l}\text { Triglycerides } \\
\text { (mM) }\end{array}$ & $\begin{array}{c}\text { Total } \\
\text { Cholesterol } \\
(\mathbf{m M})\end{array}$ & $\begin{array}{c}\text { HDL } \\
\text { Cholesterol } \\
(\mathbf{m M})\end{array}$ & $\begin{array}{c}\text { Insulin } \\
\text { (mM) }\end{array}$ \\
\hline Control & $24.0 \pm 1.7$ & $1.9 \pm 0.2$ & $1.8 \pm 0.1$ & $1.1 \pm 0.1$ & $0.36 \pm 0.03$ \\
\hline \multicolumn{6}{|l|}{ Pre-treatment } \\
\hline Diabetes & $34.9 \pm 1.7^{*}$ & $10.6 \pm 1.1^{*}$ & $3.4 \pm 0.2^{*}$ & $1.4 \pm 0.2$ & $0.07 \pm 0.02 *$ \\
\hline Diabetes + cysteine & $36.2 \pm 1.4$ & $4.2 \pm 1.3 \#$ & $2.5 \pm 0.3 \#$ & $1.3 \pm 0.1$ & $0.15 \pm 0.02 \#$ \\
\hline Diabetes + taurine & $38.2 \pm 2.1$ & $13.9 \pm 2.5$ & $3.6 \pm 0.4$ & $0.9 \pm 0.2$ & $0.11 \pm 0.03$ \\
\hline Diabetes + combination & $34.9 \pm 1.5$ & $4.1 \pm 1.4 \#$ & $2.7 \pm 0.2 \#$ & $1.6 \pm 0.2$ & $0.06 \pm 0.03$ \\
\hline \multicolumn{6}{|l|}{ Post-treatment } \\
\hline Diabetes & $35.6 \pm 1.0^{*}$ & $8.8 \pm 1.5^{*}$ & $3.1 \pm 0.2 *$ & $1.2 \pm 0.1$ & - \\
\hline Diabetes + cysteine & $31.8 \pm 1.8$ & $7.0 \pm 1.9$ & $2.5 \pm 0.3$ & $1.0 \pm 0.2$ & - \\
\hline Diabetes + taurine & $32.9 \pm 1.3$ & $12.7 \pm 2.9$ & $3.3 \pm 0.4$ & $1.1 \pm 0.2$ & - \\
\hline Diabetes + combination & $38.4 \pm 1.5$ & $8.2 \pm 1.3$ & $2.9 \pm 0.4$ & $1.2 \pm 0.2$ & - \\
\hline
\end{tabular}

Data are mean \pm SE of 5-9 animals for each group. Pre-treated and post-treated animals in each group are same as in Table 1. It should be noted that plasma levels for insulin were not determined in the post-treatment group. $* \mathrm{P}<0.05$ vs. control value; \# $\mathrm{P}<0.05$ $v s$. un-treated diabetes value.

Table 3. Echocardiographic data from diabetic rats treated with cysteine $(200 \mathrm{mg} / \mathrm{kg})$ and taurine $(400 \mathrm{mg} / \mathrm{kg})$ alone or in combination for 8 weeks.

\begin{tabular}{|c|c|c|c|c|c|c|c|}
\hline & \multirow{2}{*}{$\begin{array}{c}\text { Heart rate } \\
\text { (beats/min) }\end{array}$} & \multirow{2}{*}{$\begin{array}{c}\text { Fractional } \\
\text { shortening } \\
(\%)\end{array}$} & \multirow{2}{*}{$\begin{array}{l}\text { Cardiac } \\
\text { output } \\
\text { (1/min) }\end{array}$} & \multicolumn{2}{|c|}{ Systolic } & \multicolumn{2}{|c|}{ Diastolic } \\
\hline & & & & LViD (cm) & PWT (cm) & LViD (cm) & PWT (cm) \\
\hline $\begin{array}{l}\text { Control } \\
\text { Pre-treatment }\end{array}$ & $350 \pm 13$ & $49.1 \pm 1.5$ & $0.261 \pm 0.014$ & $0.370 \pm 0.015$ & $0.209 \pm 0.018$ & $0.730 \pm 0.011$ & $0.164 \pm 0.010$ \\
\hline $\begin{array}{l}\text { Diabetes } \\
\text { Diabetes }\end{array}$ & $282 \pm 10^{*}$ & $31.4 \pm 2.7^{*}$ & $0.172 \pm 0.012 *$ & $0.444 \pm 0.022 *$ & $0.210 \pm 0.017$ & $0.622 \pm 0.025^{*}$ & $0.192 \pm 0.018$ \\
\hline + cysteine & $324 \pm 16 \#$ & $44.7 \pm 3.9 \#$ & $0.192 \pm 0.026$ & $0.383 \pm 0.023 \#$ & $0.220 \pm 0.014$ & $0.689 \pm 0.034 \#$ & $0.137 \pm 0.004 \#$ \\
\hline+ taurine & $312 \pm 7 \#$ & $43.3 \pm 1.4 \#$ & $0.217 \pm 0.024 \#$ & $0.409 \pm 0.010 \#$ & $0.226 \pm 0.008$ & $0.725 \pm 0.029 \#$ & $0.140 \pm 0.006 \#$ \\
\hline $\begin{array}{l}+ \text { combination } \\
\text { Post-treatment } \\
\end{array}$ & $289 \pm 18$ & $45.3 \pm 2.3 \#$ & $0.176 \pm 0.015$ & $0.401 \pm 0.025 \#$ & $0.237 \pm 0.010$ & $0.710 \pm 0.015 \#$ & $0.147 \pm 0.010 \#$ \\
\hline $\begin{array}{l}\text { Diabetes } \\
\text { Diabetes }\end{array}$ & $296 \pm 14^{*}$ & $38.3 \pm 3.6^{*}$ & $0.187 \pm 0.026^{*}$ & $0.422 \pm 0.021$ & $0.234 \pm 0.014$ & $0.662 \pm 0.046$ & $0.181 \pm 0.009$ \\
\hline + cysteine & $309 \pm 21$ & $43.3 \pm 1.8$ & $0.213 \pm 0.019$ & $0.421 \pm 0.021$ & $0.218 \pm 0.011$ & $0.737 \pm 0.017$ & $0.138 \pm 0.006 \#$ \\
\hline+ taurine & $321 \pm 18$ & $44.2 \pm 1.2$ & $0.156 \pm 0.005$ & $0.351 \pm 0.023 \#$ & $0.192 \pm 0.010$ & $0.640 \pm 0.011$ & $0.139 \pm 0.003 \#$ \\
\hline+ combination & $293 \pm 18$ & $40.4 \pm 2.1$ & $0.191 \pm 0.018$ & $0.431 \pm 0.025$ & $0.211 \pm 0.015$ & $0.721 \pm 0.036$ & $0.152 \pm 0.019$ \\
\hline
\end{tabular}

Data are mean \pm SE of 5-9 animals for each group. Pre-treated and post-treated animals in each group are same as in Table 1. LViD, left ventricle internal diameter; PWT, posterior wall thickness. * $\mathrm{P}<0.05$ vs. control value; \# $\mathrm{P}<0.05$ vs. un-treated diabetic value.

animals before STZ administration (data not shown). It should be noted that the plasma glucose levels in control animals are rather high; this seems to be due to some methodological problem as plasma samples from these animals were estimated in the hospital setting. Furthermore, the glucose estimation in the plasma samples from control and experimental animals were estimated by the same methods. 
Table 4. Cardiac performance parameters of diabetic rats treated with cysteine $(200 \mathrm{mg} / \mathrm{kg})$ and taurine $(400 \mathrm{mg} / \mathrm{kg})$ alone or in combination for a period of 8 weeks.

\begin{tabular}{lcccc}
\hline & $\begin{array}{c}+\mathbf{d P} / \mathbf{d t} \\
(\mathbf{m m} \mathbf{~ H g} / \mathbf{s})\end{array}$ & $\begin{array}{c}\mathbf{- d P} / \mathbf{d t} \\
(\mathbf{m m ~ H g} / \mathbf{s})\end{array}$ & $\begin{array}{c}\text { LVSP } \\
(\mathbf{m m ~ H g})\end{array}$ & $\begin{array}{c}\text { LVEDP } \\
(\mathbf{m m ~ H g})\end{array}$ \\
\hline $\begin{array}{l}\text { Control } \\
\text { Pre-treatment }\end{array}$ & $6916 \pm 343$ & $5870 \pm 270$ & $137 \pm 9$ & $5.4 \pm 0.7$ \\
$\begin{array}{l}\text { Diabetes } \\
\text { Diabetes }+ \text { cysteine }\end{array}$ & $4644 \pm 360^{*}$ & $3218 \pm 216^{*}$ & $101 \pm 3^{*}$ & $7.0 \pm 1.8$ \\
Diabetes + taurine & $6497 \pm 192 \#$ & $4397 \pm 225 \#$ & $122 \pm 6 \#$ & $4.6 \pm 0.5$ \\
Diabetes + combination & $5823 \pm 293 \#$ & $4248 \pm 319 \#$ & $108 \pm 4$ & $6.4 \pm 0.7$ \\
Post-treatment & $5680 \pm 234 \#$ & $3862 \pm 237 \#$ & $101 \pm 4$ & $5.6 \pm 0.5$ \\
$\begin{array}{l}\text { Diabetes } \\
\text { Diabetes }+ \text { cysteine }\end{array}$ & $5835 \pm 135^{*}$ & $4160 \pm 305^{*}$ & $107 \pm 7^{*}$ & $4.4 \pm 0.8$ \\
Diabetes + taurine & $5991 \pm 195$ & $4022 \pm 312$ & $113 \pm 9$ & $6.5 \pm 0.7$ \\
Diabetes + combination & $5432 \pm 344$ & $3765 \pm 129$ & $107 \pm 7$ & $8.1 \pm 1.7$ \\
\hline
\end{tabular}

Data are mean \pm SE of 5-9 animals for each group. Pre-treated and post-treated animals in each group are same as in Table $1 .+\mathrm{dP} / \mathrm{dt}$ rate of contraction; -dP/dt, rate of relaxation; LVSP, left ventricle systolic pressure; LVEDP, left ventricle end diastolic pressure. * $\mathrm{P}<0.05$ vs. control value; \# $\mathrm{P}<0.05$ vs. un-treated diabetes value.

Echocardiographic and cardiac performance assessment of diabetic animals treated with cysteine and taurine

The HR, FS and CO were significantly reduced in the diabetic rats. In addition, while an increase in LViDs was observed, LViDd was decreased in diastole in the diabetes group. Although the mean values for PWT in diastole, unlike systole, of the diabetic heart were higher than control values, statistical significance was not reached (Table 3 ). In the prevention arm, both cysteine and taurine prevented the changes in HR, FS, LViDs, LViDd and PWT in diastole. However, the effects of cysteine and taurine on these parameters were not addititive. Although taurine, unlike cysteine, also prevented the depression in $\mathrm{CO}$, pre-treatment of diabetic animals with combination of taurine and cysteine did not affect this parameter (Table 3). Treatment of the diabetic animals for reversal with cysteine, taurine or in combination did not affect diabetes-induced changes in cardiac performance or cardiac remodeling except that changes in PWT in diastole were attenuated by both cysteine and taurine whereas those in LViDs and LViDd were affected by taurine only (Table 3 ). Diabetes was also found to decrease left ventricle systolic pressure (LVSP) and reduce rate of contraction $(+\mathrm{dP} / \mathrm{dt})$ and rate of relaxation $(-\mathrm{dP} / \mathrm{dt})$ without any change in left ventricle end diastolic pressure (LVEDP). In the prevention protocol, unlike the reversal protocol, cysteine and taurine alone or in combination were found to attenuate the diabetes-induced changes in $+\mathrm{dP} / \mathrm{dt}$ and $-\mathrm{dP} / \mathrm{dt}$ (Table 4). Pre-treatment with cysteine, unlike taurine, was also observed to attenuate diabetes-induced changes in LVSP. Combination of cysteine and taurine did not produce any addititive effect (Table 4).

\section{Discussion}

In agreement with our earlier studies diabetes was associated with depressed cardiac function, increased HW/BW ratio and elevated levels of plasma glucose as well as triglycerides and cholesterol (Dhalla et al. 1998, Tappia et al. 2004, 2011). Furthermore, cardiac dysfunction was associated with $\mathrm{LV}$ remodeling because PWT and LV internal diameters (LViDs and LViDd) were altered due to diabetes. Interestingly, HW/BW ratio, which is considered to be index of cardiac hypertrophy, was increased as a consequence of the reduced BW in diabetic animals. The present study was undertaken to assess the efficacy of cysteine alone or in combination with taurine for prevention and reversal. The salient findings were that cysteine in the prevention arm (a) like taurine, prevented cardiac dysfunction in diabetes; (b) unlike taurine, reduced plasma level of triglycerides; (c) cysteine with or without taurine prevented diabetesinduced alterations in LViDs; (d) cysteine in combination with taurine did not exert any additive effects; (e) in the reversal arm, treatment of the diabetic animals with 
cysteine alone or in combination with taurine did not reverse the alterations in hemodynamic parameters, hyperglycemia or lipid abnormalities. These observations support the view that both cysteine and taurine prevent the diabetes-induced alterations in cardiac function, but these amino acids do not exert any addititive actions. Indeed, it seems that the protective effects of both amino acids administered separately are linearly dose-dependent while excessive intake of these amino acids has no beneficial effects and could even produce toxic effects.

The sustained elevation in blood glucose is associated with low cellular levels of GSH (Wu et al. 2004, Sekhar et al. 2011) leading to oxidative stress and subsequent diabetic cardiomyopathy (Dhalla et al. 1998). In fact, in human diabetic erythrocyte concentrations of cysteine and GSH have been reported to be significantly lower than in control subjects (Sekhar et al. 2011). While cysteine supplementation has been reported to lower insulin resistance, glycemia and oxidative stress in Zucker diabetic fatty rats (Jain et al. 2009), the present study is the first to report the cardioprotective effects of cysteine in STZ-induced diabetes rat model. Although we did not determine oxidative stress and cellular GSH levels, we believe that cysteine is acting as antioxidant since cysteine is known to be an effective oxidant scavenger (Tang et al. 1991). In addition an indirect effect through GSH may also be involved in the beneficial actions of cysteine (Shackebaei et al. 2005).

It is interesting to note that $\gamma$-amino butyric acid has been reported to restore pancreatic $\beta$-cell mass and reverse diabetes; such effects on $\beta$-cell regeneration and regulation of glucose homeostasis may have clinical application for this particular amino acid in diabetes (Soltani et al. 2011). In the present study, we observed a significant increase in plasma levels of insulin in the cysteine treated diabetic animals, however; this did not translate into reduced hyperglycemia. In this regard, it can be suggested that while some protection of pancreatic $\beta$-cell function may have been obtained with cysteine, the improvement in insulin production may have been below the threshold levels to have impact on hyperglycemia. It is difficult for us to elucidate the mechanisms of partial augmentation of insulin level by cysteine and the effect of cysteine on having a direct effect on the potency of STZ as it requires extensive studies, however; $\beta$-cell regeneration in the diabetic animals cannot be ruled out. Furthermore, since the effects of cysteine on insulin levels in the prevention arm did not translate into hypoglycemic action, it can be argued that there is a conversion of type 1 diabetes into type 2 diabetes under our experimental conditions. However, this view cannot be substantiated because we did not determine plasma insulin level in the reversal arm. At the same time, it would be interesting to also measure $\mathrm{Hb} 1 \mathrm{Ac}$ to better understand the impact of cysteine on blood glucose levels. Thus, the possibility arises that a longer treatment period or administration of higher dose of cysteine may have exerted greater effects on glucose metabolism.

The view that the beneficial effects of cysteine may partly be due to the protection of $\beta$-cells and subsequent increase in the plasma level of insulin, is supported by our observation that improvement of cardiac function with cysteine was seen in the prevention arm and not in the reversal arm of the study. Taken together, the beneficial actions of cysteine may be not only be related to antioxidant effects, but also due to increase in plasma insulin levels; this possibility warrants further confirmation. However, it is pointed out that while the focus of the present study was to investigate the ability of cysteine alone or in combination with taurine to prevent or reverse the cardiovascular complications due to diabetes rather than for prevention of diabetes per se, these findings are suggestive of a therapeutic potential of cysteine in patients diagnosed with type 1 diabetes.

In the reversal protocol neither cysteine and taurine alone nor the combination of cysteine and taurine resulted in any recovery in $+\mathrm{dP} / \mathrm{dt}$ and $-\mathrm{dP} / \mathrm{dt}$. In fact, the effect of the combination did not differ significantly from the single treatments. These observations may be related to a limited ability to reverse diabetes-induced oxidative damage with these amino acids. Support for this contention is indicated by the fact that in the prevention protocol cysteine and taurine alone as well as in combination either completely or partially prevented the depressions in $+\mathrm{dP} / \mathrm{dt}$ and $-\mathrm{dP} / \mathrm{dt}$ due to diabetes.

We have previously reported the potential mechanisms for beneficial action of taurine on the heart during diabetes (Xu et al. 2008, Tappia et al. 2011). While cysteine may be cytoprotective also through its antioxidant activity, some other mechanisms of beneficial action of cysteine can be proposed. The absence of an additive effect of cysteine with taurine suggests a common mechanism of action; however, since there is an overlap on the beneficial effects of cysteine and taurine on cardiac function in diabetes, other mechanisms to explain the beneficial actions of cysteine, specifically with regard to the triglyceride and total cholesterol lowering effect during diabetes need to be determined. It 
is well known that the reduced contractile performance during diabetes is associated with abnormal $\mathrm{Ca}^{2+}$ handling and oxidative stress (Dhalla et al. 1998). Accordingly, it could be suggested that the beneficial effects of cysteine may be linked to improved cardiomyocyte $\mathrm{Ca}^{2+}$ - homeostasis and antioxidant status. While taurine has been reported to increase $\left[\mathrm{Ca}^{2+}\right]_{i}$ and the amplitude of the $\mathrm{Ca}^{2+}$-transient in diabetic cardiomyocytes (Holloway et al. 1999), cysteine has been reported to prevent the increase in oxidant-induced increase in $\left[\mathrm{Ca}^{2+}\right]_{\mathrm{i}}$ in rat ventricular cardiomyocytes (Fukui et al. 1994). On the other hand, hydrogen sulfide, which is produced endogenously from cysteine in mammalian tissues (Lee et al. 2006) has been reported to increase $\left[\mathrm{Ca}^{2+}\right]_{\mathrm{i}}$ through $\mathrm{Ca}^{2+}$ from intracellular stores and increases in $\mathrm{Ca}^{2+}$-influx across the plasma membrane in micoglial cells (Lee et al. 2006). Thus it is possible that cysteine may indirectly regulate $\left[\mathrm{Ca}^{2+}\right]_{i}$ in diabetic cardiomyocytes and improve $\mathrm{Ca}^{2+}$-handling in diabetic cardiomyocytes. It is also possible that the beneficial actions of cysteine may be due to a metabolic control rather than their utilization. These potential mechanisms of action of cysteine therefore need to be investigated further.

$\mathrm{N}$-acetylcysteine (NAC), a commercially available form of cysteine, is known to attenuate high glucose-induced cardiomyocyte death (Fiordaliso et al.
2004, Kumar and Sitasawad 2009). However, NAC is readily converted to cysteine, in vivo, and thus the beneficial effects of NAC may also be attributable to increased formation of cysteine. While our study has focussed on type 1 diabetes, extension of our hypothesis to the type 2 disease is warranted since $>90 \%$ of the diabetic population are of this type. Nonetheless, in conclusion, our findings suggest that cysteine either alone or in combination with taurine may prevent diabetes induced cardiac abnormalities as well as increases in triglyceride levels. However, the data do not support use of cysteine alone or in combination with taurine as a therapeutic intervention for reversal of cardiac dysfunction and elevated triglyceride levels.

\section{Conflict of Interest}

There is no conflict of interest.

\section{Acknowledgements}

This study was supported by a Research Grant form CheemoCree Pharma. Inc., Winnipeg, Canada. Infrastructural support was provided by St. Boniface Hospital Research Foundation. D. R.-L. was a Visiting Scientist from Cardiovascular Research Division, V. I. Lenin University Hospital, Holguin, Cuba, and was supported by the Heart and Stroke Foundation of Canada.

\section{References}

BAE SK, YANG SH, KIM JW, KIM T, KWON JW, LEE MG: Effects of cysteine on the pharmacokinetics of olitipraz in rats with protein-calorie malnutrition. J Pharm Sci 94: 1484-1493, 2005.

BOUCKENOOGHE T, REMACLE C, REUSENS B: Is taurine a functional nutrirent? Curr Opin Clin Nutr Metab Care 9: 728-733, 2006.

DHALLA NS, LIU X, PANAGIA V, TAKEDA N: Subcellular remodeling and heart dysfunction in chronic diabetes. Cardiovasc Res 40: 239-247, 1998.

DU Y, MILLER CM, KERN TS: hyperglycemia increases mitochondrial superoxide in retina and retinal cells. Free Radic Biol Med 35: 1491-1499, 2003.

FUKUI K, KANEDA M, TAKAHASHI E, WASHIO M, DOI K: Protective effects of sulfhydryl compounds on HOClinduced intracellular $\mathrm{Ca}^{2+}$ increase in single rat ventricular myocytes. J Mol Cell Cardiol 26: 455-461, 1994.

GRIFFITH OW: Biologic and pharmacologic regulation of mammalian glutathione synthesis. Free Rad Biol Med 27: 922-935, 1999.

HANSEN SH: The role of taurine in diabetes and the development of diabetic complications. Diabetes Metab Res Rev 17: 330-346, 2001.

HOLLOWAY C, KOTSANAS G, WENDT I: Acute effects of taurine on intracellular calcium in normal and diabetic cardiac myocytes. Pflugers Arch 438: 384-391, 1999.

JAIN SK, VELUSAMY T, CROAD JL, RAINS JL, BULL R: L-cysteine supplementation lowers blood glucose, glycated hemoglobin, CRP, MCP-1, and oxidative stress and inhibits NF- $\mathrm{KB}$ activation in the livers of Zucker diabetic rats. Free Radic Biol Med 46: 1633-1638, 2009. 
KUMAR S, SITASAWAD SL: N-acetylcysteine prevents glucose/glucose oxidase-induced oxidative stress, mitochondrial damage and apoptosis in H9c2 cells. Life Sci 84: 328-336, 2009.

LEE SW, HU YS, HU LF, LU Q, DAWE GS, MOORE PK, WONG PT, BIAN JS: Hydrogen sulphide regulates calcium homeostasis in microglial cells. Glia 54: 116-124, 2006.

MEISTER A, ANDERSON ME, HWANG O: Intracellular cysteine and glutathione delivery systems. J Am Coll Nutr 5: 137-151, 1986.

O'CONNOR E, DEVESA A, GARCIA C, PUERTES IR, PELLIN A, VINA JR: Biosynthesis and maintenance of GSH in primary astrocyte cultures: role of l-cysteine and ascorbate. Brain Res 680: 157-163, 1995.

SEKHAR RV, MCKAY SV, PATEL SG, GUTHIKONDA AP, REDDY VT, BALASUBRAMANYAM A, JAHOOR F: Glutathione synthesis is diminished in patients with uncontrolled diabetes and restored by dietary supplementation with cysteine and glycine. Diabetes Care 34: 162-167, 2011.

SHACKEBAEI D, KING N, SHUKLA B, SULEIMAN M-S: Mechanisms underlying the cardioprotective effect of 1-cysteine. Mol Cell Biochem 277: 27-31, 2005.

SOLTANI N, QIU H, ALEKSIC M, GLINKA Y, ZHAO F, LIU R, LI Y, ZHANG N, CHAKRABARTI, R, NG T, JIN T, ZHANG H, LU WY, FENG ZP, PRUD'HOMME GJ, WANG Q: GABA exerts protective and regenerative effects on islet $\beta$ cells and reverses diabetes. Proc Natl Acad Sci USA 108: 11692-11697, 2011.

STIPANUK MH, COLOSSO RM, GARCIA RA, BANKS MF: Cysteine concentration regulates cysteine metabolism to glutathione, sulfate and taurine in rat hepatocytes. J Nutr 122: 420-427, 1992.

TANG L-D, SUN J-Z, WU K, SUN C-P, TANG Z-M: Beneficial effects of N-acetylcysteine and cysteine in stunned myocardium in perfused rat heart. Br J Pharmacol 102: 601-606, 1991.

TAPPIA PS, ASEMU G, AROUTIOUNOVA N, DHALLA NS: Defective sarcolemmal phospholipase C signaling in diabetic cardiomyopathy. Mol Cell Biochem 261: 193-199, 2004.

TAPPIA PS, THLIVERIS J, XU YJ, AROUTIOUNOVA N, DHALLA NS: Effects of amino acid supplementation on myocardial cell damage and cardiac function in diabetes. Exp Clin Cardiol 16: e17-e25, 2011.

WU G, FANG YZ, YANG S, LUPTON JR, TURNER ND: Glutathione metabolism and its implications for health. J Nutr 134: 489-492, 2004.

XU YJ, ARNEJA AS, TAPPIA PS, DHALLA NS: The potential health benefits of taurine in cardiovascular disease. Exp Clin Cardiol 13: 57-65, 2008. 